Case report

\title{
Adjunctive use of antimicrobial photodynamic therapy in the treatment of medication-related osteonecrosis of the jaws: A case report
}

\author{
Pier Paolo Poli ${ }^{\mathrm{a}, *}$, Francisley Ávila Souza ${ }^{\mathrm{b}}$, Carlo Maiorana ${ }^{\mathrm{a}}$ \\ a Implant Center for Edentulism and Jawbone Atrophies, Maxillofacial Surgery and Odontostomatology Unit, Maggiore Policlinico Hospital, University of Milan, Via \\ Commenda 10, 20122 Milan, Italy \\ b Department of Surgery and Integrated Clinic, Araçatuba Dental School, São Paulo State University “Júlio de Mesquita Filho" - UNESP, Rua José Bonifácio, 1193 - Vila \\ Mendonca, Araçatuba, SP, 16015-050, Brazil
}

\section{A R T I C L E I N F O}

\section{Keywords:}

Bisphosphonates

Decontamination

Photodynamic therapy

Oral surgery

Osteonecrosis

\begin{abstract}
A B S T R A C T
Medication-related osteonecrosis of the jaws is a delayed healing condition commonly recognized as a serious adverse effect of antiresorptive therapy and is particularly associated with aminobisphosphonate treatment. In the present report, a 62-year-old osteoporotic female patient treated with intramuscular injections of clodronate for 3 years exhibited clinical signs of exposed necrotic bone and inflamed mucosa 4 months after tooth extractions in the mandibular interforaminal region. The treatment consisted of sequestrectomy, open-flap debridement, and adjunctive use of antimicrobial photodynamic therapy to decontaminate the affected hard and soft tissues. Successful results were obtained and maintained from both clinical and radiological aspects. No recurrence was observed up to 6 months of follow-up.
\end{abstract}

\section{Introduction}

Antiresorptive agents and angiogenesis inhibitors are commonly administered to the osteoporosis patient population. Despite numerous advantages, bisphosphonates and human monoclonal antibodies have been associated with medication-related osteonecrosis of the jaws (MRONJ) in osteoporotic patients who underwent oral surgery procedures [1]. In addition to the intake of antiresorptive or antiangiogenic agents, MRONJ is characterized by exposed bone or bone that can be probed through intraoral or extraoral fistulae in the maxillofacial region that has persisted for more than 8 weeks, with no history of radiation therapy or obvious metastatic disease of the jaws [2]. Antibiotic prophylaxis is an essential preventive measure to avoid the onset of osteonecrosis in patients scheduled for tooth extraction and/or dental implant removal who are currently being treated with medications implicated in the development of MRONJ. However, the presence of bacteria in combination with fungi and viruses as important components of the osteonecrosis-related multi-organism biofilm has prompted studies to evaluate more sophisticated therapies. Antimicrobial photodynamic therapy (aPDT), which contemplates the interaction between a non-thermal light source of a specific wavelength $(630-880 \mathrm{~nm})$ and a photosensitizer, has led to several dental applications with promising results, including the treatment of MRONJ [3]. On the other hand, the use of aPDT for the treatment of MRONJ remains underreported. Hence, the purpose of the present paper was to report the use of aPDT for the treatment of MRONJ in a patient treated with bisphosphonates.

\section{Case report}

A 62-year-old non-smoking Caucasian woman was referred for the evaluation of exposed bone in the buccal vestibule of the mandible. During anamnesis, the patient reported the extraction of the inferior teeth 4 months prior, followed by alveolar mucosal inflammation and pain thereafter. Her medical history included osteoporosis treated with the intramuscular injection of clodronate $100 \mathrm{mg}$ /week for 3 years. Intraoral examination revealed no signs of primary wound healing, with two portions of exposed necrotic bone under dehiscent mucosa located in the vestibular interforaminal region of the mandible with no evidence of infection (Fig. 1A). The case history along with the clinical findings fulfilled the criteria for MRONJ stage 1 according to the AAOMS staging [2]. Medications prescribed consisted of the oral administration of amoxicillin $1 \mathrm{~g}$ every $8 \mathrm{~h}$ for 20 days starting three days before the surgical procedure, and mouth rinsing with $0.2 \%$ chlorhexidine twice daily for the same period. Post-operative pain was controlled with ibuprofen $600 \mathrm{mg}$ administered every $8 \mathrm{~h}$ for 3 days. Surgery was performed on an outpatient basis under local anesthesia.

\footnotetext{
* Corresponding author at: Implant Center for Edentulism and Jawbone Atrophies, Maxillo-Facial Surgery and Odontostomatology Unit, Fondazione Cà Granda IRCCS, Ospedale Maggiore Policlinico, University of Milan, Via Commenda 10, 20122 Milan, Italy.

E-mail addresses: pierpaolo.poli@unimi.it (P.P. Poli), f.avilasouza@foa.unesp.br (F.Á. Souza), carlo.maiorana@unimi.it (C. Maiorana).
} 

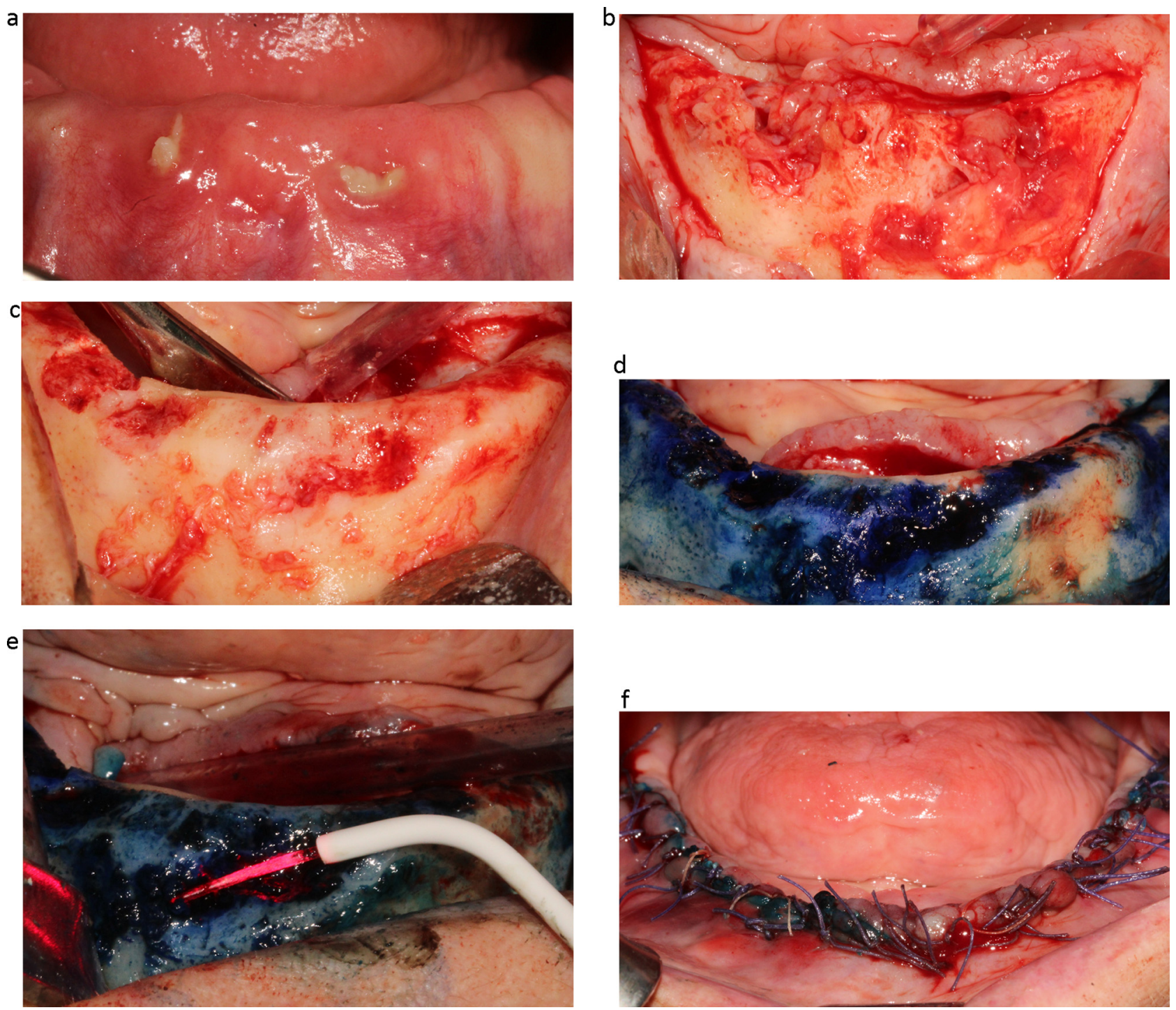

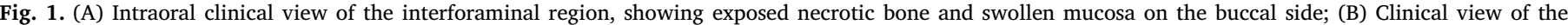

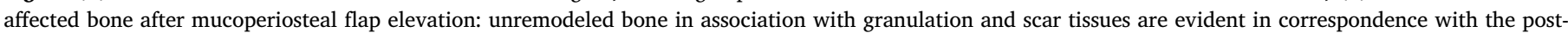

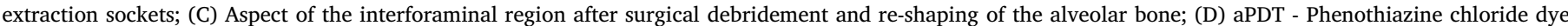
injected topically on the surgical site; (E) aPDT - Irradiation of the dyed surface with a 660 nm diode laser; (F) Tension-free suture of the surgical flaps.

Briefly, a full-thickness flap was raised and the extraction sockets were meticulously debrided and curetted to remove all granulation and infected tissues (Fig. 1B). Sequestrectomy of the exposed bone and recontouring of the residual ridge were performed using pear-shaped burs mounted on a surgical handpiece under copious irrigation with sterile saline (Fig. 1C). Once the alveolar bone was noted to be bleeding favorably, aPDT was performed to minimize the local infection and to biostimulate the soft tissues with a specific setup (HELBO ${ }^{\circ}$, Bredent Medical, Senden, Germany). A $0.5-\mathrm{mL}$ solution of $10 \mathrm{mg} / \mathrm{mL}$ phenothiazine chloride dye consisting of Methylenthioniniumchlorid (HELBO ${ }^{\circ}$ Blue Photosensitizer, Bredent Medical, Senden, Germany) based on methylene blue compound, was applied over the affected region and left in place for $3 \mathrm{~min}$ (Fig. 1D). Subsequently, the area was rinsed vigorously for $1 \mathrm{~min}$ with sterile saline to remove the excess photosensitizer. A hand-held $100 \mathrm{~mW}$ diode laser with a wavelength of $660 \pm 10 \mathrm{~nm}\left(\mathrm{HELBO}^{\circ}\right.$ TheraLite Laser, Bredent Medical, Senden, Germany) equipped with a dedicated probe (HELBO ${ }^{\circ}$ 3D Pocket Probe, Bredent Medical, Senden, Germany) providing a power density of $60 \mathrm{~mW} / \mathrm{cm}^{2}$ was used to activate the previously dyed surface of approximately $3 \mathrm{~cm}^{2}$ for $60 \mathrm{~s}$ per $\mathrm{cm}^{2}$ with circular movements (Fig. 1E).
The resulting fluence was $3.6 \mathrm{~J} / \mathrm{cm}^{2}$, while the total energy applied was $10.8 \mathrm{~J}$. After irradiation, the entire region was rinsed with sterile saline. Finally, periosteal releasing incisions were performed to advance the flaps coronally and a tension-free suture was applied with horizontal mattress sutures and single knots using absorbable suture material (4-0 and 5-0 Vicryl, Ethicon Inc., Somerville, NJ, USA) (Fig. 1F). Soft tissue healing was promoted with weekly applications of low-level laser therapy (LLLT) with the same apparatus for 6 weeks. The sutures were removed after 2 weeks, and recall visits were scheduled weekly for the first 45 days and monthly thereafter for 6 months. The healing proceeded uneventfully. A complete resolution of the disease was observed in terms of the maintenance of mucosal closure without any signs of residual infection, fistulae, or exposed necrotic bone at the surgical site (Fig. 2A). Clinical healing was corroborated by a radiological evaluation showing no radiographic signs of MRONJ and smooth margins at the 6-month follow-up (Fig. 2B).

\section{Discussion}

The outcome of the present case report reinforces the use of aPDT 


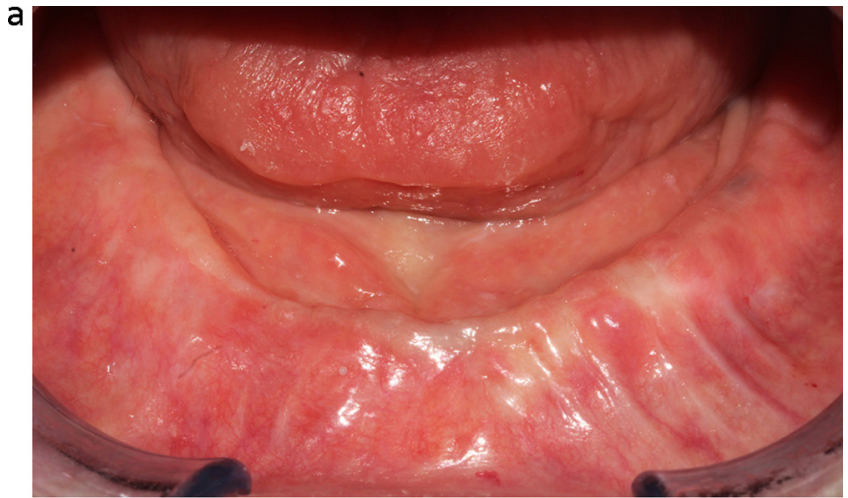

b

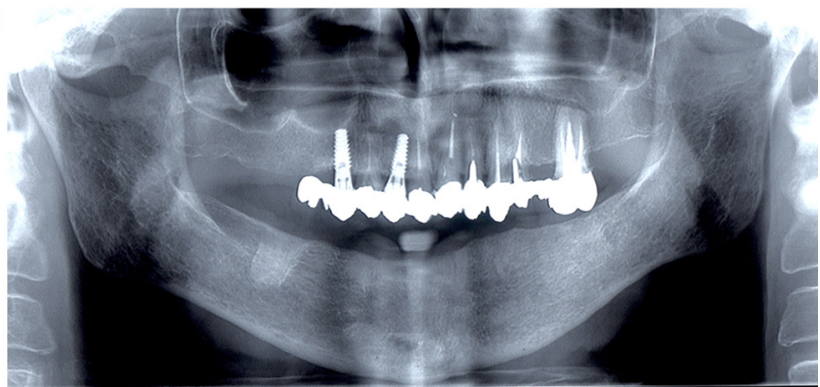

Fig. 2. (A) Intraoral clinical view of the soft tissues healing at the 6-month follow-up recall, showing first intention healing of the surgical wound, with no clinical signs of exposed bone and mucosal inflammation; (B) Post-operative 6month orthopantomograph: no radiological signs of MRONJ are visible, with physiologically healed and well-defined bone tissue.

and LLLT for the successful treatment of MRONJ. This was already noted in previous reports on MRONJ [3,4]. The advantages of laserassisted treatments are known, including decontamination and photobiostimulation that accelerates disease resolution and soft tissue healing. These characteristics become essential when the empiric use of local and/or systemic antibiotics might be insufficient to eradicate the complex biofilm formation on the exposed bone of MRONJ. For this reason, in the case presented herein, aPDT was used as adjuvant therapy in the eradication of the pathogenic biofilm. Gram-positive and gram-negative bacterial pathogens, as well as parasites, fungi, and viruses identified in MRONJ lesions, are the targets of the reactive oxygen species released during the photochemical process of the aPDT. In particular, Actinomyces species are among the major contributors to biofilm formation in patients with osteonecrosis [2]. Interestingly, phenothiazine chloride irradiated with a $660 \mathrm{~nm}$ wavelength $100 \mathrm{~mW}$ low-power continuous-wave diode laser has shown the most effective bactericidal activity against Actinomyces naeslundii compared to chlorhexidine and polyhexanide in vitro [5]. It is noteworthy that the same dye consisting of $1 \%$ methylene blue (3.7-bis-(dimethylamino)-phenothiazin-5-ium chloride), glucose, methylhydroxypropylcellulose, and citrate was applied in the present report with successful results. The same applies to Staphylococcus aureus, which is responsible for most bone infections and is effectively inactivated using aPDT in vitro and in vivo [6].

The role of clodronate, a non-nitrogen-containing bisphosphonate, in the etiopathogenesis of the osteonecrosis in the present report is noteworthy. Indeed, clodronate has been shown to alter the physiologic processes and impair the repair capacity of osteoblasts, decreasing the gene expression of molecules directly related to cell maturation, which could explain the onset of MRONJ [7]. A fortiori, aPDT with phenothiazine chloride was used in view of the absence of a negative effect on the growth and differentiation of human osteoblastic cells without concomitantly counteracting the biostimulatory effect induced by LLLT [8]. The beneficial effects of aPDT also extend to the soft tissue cells due to the cold photochemical process of the therapy itself. Connective tissues such as collagen and elastin are unaffected, and the proliferation, attachment, and collagen synthesis of gingival fibroblasts are stimulated [9]. The promotion of soft tissue regeneration was exploited in this clinical case, where the primary wound closure of the surgical site played a pivotal role in the success of the treatment.

The risk of developing MRONJ associated with oral bisphosphonates increases when the duration of therapy exceeds 4 years [2]. However, even if the duration of treatment with alkylbiphosphonates before MRONJ occurrence is longer, the time of onset after the beginning of bisphosphonate therapy has been reported to be sometimes less than 4 years [10]. Accordingly, in the present report, the patient was administered clodronate for 3 years, with the first clinical signs of osteonecrosis visible 4 months after tooth extractions. Hence, although the bisphosphonates most frequently related to MRONJ contain nitrogen, osteonecrosis induced by the administration of alkylbiphosphonates should not be underestimated.

\section{Declarations of interest}

None declared.

\section{Funding sources}

This research did not receive any specific grant from funding agencies in the public, commercial, or not-for-profit sectors.

\section{References}

[1] A.A. Khan, A. Morrison, D.A. Hanley, et al., Diagnosis and management of osteonecrosis of the jaw: a systematic review and international consensus, J. Bone Miner. Res. 30 (2015) 3-23.

[2] S.L. Ruggiero, T.B. Dodson, J. Fantasia, et al., American association of oral and maxillofacial surgeons position paper on medication-related osteonecrosis of the jaw-2014 update, J. Oral Maxillofac. Surg. 72 (2014) 1938-1956.

[3] M.S. de Castro, N.V. Ribeiro Jr., M.L. de Carli, et al., Photodynamically dealing with bisphosphonate-related osteonecrosis of the jaw: successful case reports, Photodiagn. Photodyn. Ther. 16 (2016) 72-75.

[4] P. Vescovi, I. Giovannacci, E. Merigo, et al., Tooth extractions in high-risk patients under bisphosphonate therapy and previously affected with osteonecrosis of the jaws: surgical protocol supported by low-level laser therapy, J. Craniofac. Surg. 26 (2015) 696-699.

[5] S. Hafner, M. Ehrenfeld, E. Storz, et al., Photodynamic inactivation of actinomyces naeslundii in comparison with chlorhexidine and polyhexanide-a new approach for antiseptic treatment of medication-related osteonecrosis of the jaw? J. Oral Maxillofac. Surg. 74 (2016) 516-522.

[6] S.K. Bisland, C. Chien, B.C. Wilson, et al., Pre-clinical in vitro and in vivo studies to examine the potential use of photodynamic therapy in the treatment of osteomyelitis, Photochem. Photobiol. Sci. 5 (2006) 31-38.

[7] F.J. Manzano-Moreno, J. Ramos-Torrecillas, E. De Luna-Bertos, et al., Effect of clodronate on antigenic profile, growth, and differentiation of osteoblast-like cells, J. Oral Maxillofac. Surg. 74 (2016) 1765-1770.

[8] E. Stein, J. Koehn, W. Sutter, et al., Phenothiazine chloride and soft laser light have a biostimulatory effect on human osteoblastic cells, Photomed. Laser Surg. 27 (2009) 71-77.

[9] J. Qiao, S. Wang, Y. Wen, et al., Photodynamic effects on human periodontal-related cells in vitro, Photodiagn. Photodyn. Ther. 11 (2014) 290-299.

[10] S. Crepin, M.L. Laroche, B. Sarry, et al., Osteonecrosis of the jaw induced by clodronate, an alkylbiphosphonate: case report and literature review, Eur. J. Clin. Pharmacol. 66 (2010) 547-554. 